\title{
Quaderni
}

QUADERN I Communication, technologies, pouvoir

77 | Hiver 2011-2012

L'évaluation de la recherche

\section{Entre qualité prescrite et qualité souhaitable}

L'ambivalence des chercheurs en communication face à l'évaluation de leurs publications

Marc Vanholsbeeck

\section{(2) OpenEdition}

Édition électronique

URL : http://journals.openedition.org/quaderni/554

DOI : 10.4000/quaderni.554

ISSN : 2105-2956

Éditeur

Les éditions de la Maison des sciences de l'Homme

Édition imprimée

Date de publication : 5 janvier 2012

Pagination : 71-84

Référence électronique

Marc Vanholsbeeck, "Entre qualité prescrite et qualité souhaitable », Quaderni [En ligne], 77 | Hiver

2011-2012, mis en ligne le 05 janvier 2014, consulté le 19 avril 2019. URL : http://

journals.openedition.org/quaderni/554; DOI : 10.4000/quaderni.554 


\section{$D$ ossier}

entre qualité prescrite et qualité souhaitable L'ambivalence des chercheurs en communication face à l'évaluation de leurs publications

\section{Marc Vanholsbeeck}

Assistant-chercheur Département des Sciences de

l'information et de la communication Université Libre de Bruxelles
D'importants bouleversements affectent l'univers professionnel de l'enseignement supérieur. Les universités, plus autonomes et « responsables », se livrent à une concurrence internationale, structurée sur le mode du benchmarking par la publication de nombreux classements. La place qu'y occupera un établissement dépend désormais largement de son degré d'internationalisation, ainsi que de sa productivité en articles scientifiques. Pour intégrer ces transformations fondamentales à la gestion des universités et de leurs ressources humaines, les principes du "nouveau management public » sont de plus en plus utilisés ${ }^{1}$, qui engagent à renoncer au contrôle direct des processus de fonctionnement de la recherche pour privilégier des modes de pilotage à distance de cette dernière. En particulier, des instruments d'évaluation des différents types de «produits» académiques - et, en premier lieu, des publications scientifiques - sont mis en place. Le corollaire en sera le recours au concept clé, polymorphe et surplombant, de la «qualité », définissant les caractéristiques des performances à fournir en matière d'enseignement et de recherche ${ }^{2}$.

Si la littérature a bien abordé ces évolutions aux niveaux macro et institutionnel ${ }^{3}$, on a beaucoup moins analysé comment les chercheurs perçoivent et s'approprient, au quotidien, les exigences de qualité relatives à l'évaluation de leurs publications ${ }^{4}$. À cet égard, le cas des chercheurs en sciences humaines et sociales (SHS) actifs dans l'espace francophone mérite un intérêt particulier, ainsi que la problématique de leur degré de (re) connaissance et d'incorporation des prescriptions, des recherches spécifiques. D'une part, les SHS 
francophones sont exposées depuis peu à des systèmes de mesure de la qualité de type scientométrique - tel par exemple le Thomson Reuters' Web of Science -, fondés sur le calcul des citations, et conçus initialement dans le monde anglo-saxon afin d'évaluer les disciplines scientifiques, techniques et mathématiques (STM). D'autres tentatives, développées en tant qu'alternatives plus respectueuses des spécificités des SHS - mais pas moins polémiques pour autant - ont visé à l'établissement, par des comités d'experts, de listes de revues qualifiantes, hiérarchisées ou non, telles les listes françaises de l'AERES (Agence d'évaluation de la recherche et de l'enseignement supérieur) ou celles de l'ESF (European Science Foundation) à l'échelle européenne ${ }^{5}$.

D'autre part, la valorisation professionnelle des chercheurs en SHS passe de plus en plus par l'évaluation de la portée internationale de leurs travaux, alors que l'internationalité est, de manière générale, moins ancrée dans leurs traditions disciplinaires que dans celles des $\mathrm{STM}^{6}$. Par conséquent, l'actuelle reformulation des critères de la qualité dans l'évaluation des publications est régulièrement décriée, au sein de l'espace professionnel de la recherche francophone en SHS. En particulier, elle manifesterait des velléités de mise au pas des chercheurs par le pouvoir politique, ainsi qu'une emprise culturelle croissante de la recherche anglo-saxonne et des méthodes quantitatives d'évaluation. Elle impliquerait également la dévalorisation des revues nationales non anglophones, ainsi que celle des écoles de pensée qui y auraient trouvé un lieu de développement privilégié7.

Dans ce contexte, il importe de mieux fonder la critique des dispositifs d'évaluation sur le recueil et l'analyse scientifique de données de terrain. Partant, notre objectif sera ici de questionner empiriquement les trois présupposés suivants, sous-jacents à bien des débats actuels au sein de la recherche francophone : celui du caractère formalisé et explicite des prescriptions en matière d'évaluation de la qualité des publications en SHS, celui de la dichotomie des attitudes - adhésion vs résistance - manifestées par les chercheurs à leur égard et, enfin, celui d'un impact mécanique et unilatéral de l'évaluation sur les pratiques de dissémination scientifique.

En nous fondant sur l'analyse exploratoire d'une communauté géographique et disciplinaire particulière - celle des chercheurs en sciences de la communication de Belgique francophone -, nous soutiendrons ici la thèse que la qualité dans l'évaluation des publications n'est pas (encore) une notion «qui va de soi » pour les chercheurs, mais qu'elle est plutôt à l'origine de représentations foncièrement ambivalentes, ainsi que d'un mode d'intégration composite du prescrit aux pratiques de publication.

\section{Méthodologie et spécificités du terrain}

Douze entretiens compréhensifs approfondis ont été réalisés entre novembre 2010 et avril 2011, avec des chercheurs affiliés à des unités de recherche en sciences de la communication d'institutions universitaires belges francophones. Ce terrain présente une hétérogénéité particulièrement propice au recueil d'une variété optimale de représentations relatives à la qualité et pose, dans l'optique de notre problématique, les sciences de la communication belges franco- 
phones en véritable microcosme des SHS. Tout d'abord, les unités de recherche en communication rassemblent, en effet, du personnel issu d'horizons disciplinaires divers : à côté de jeunes chercheurs doctorants dans la discipline, on y trouve également des docteurs diplômés d'autres secteurs des SHS (histoire, philologie romane, mais également : droit, sciences de la documentation ou ingénierie de gestion). Or, les affinités historiques à l'usage de la langue anglaise et à la discussion internationale des résultats, voire la proximité avec les modes de publication des STM - i.e. : articles courts et collectifs rendant compte de découvertes ponctuelles plutôt que monographies longuement mûries, reflétant le cheminement d'une pensée individuelle-, varient fortement entre ces disciplines. Ensuite, même si leurs traditions sont encore jeunes, les sciences de la communication belges francophones se situent elles-mêmes, en tant qu'inter-disciplines, à la croisée des sciences de l'information et de la communication à la française (SIC) et des Communication Studies anglo-saxonnes, avec des affinités sélectives envers les unes ou les autres, qui sont propres à l'histoire spécifique des départements et des unités de recherche au sein de chaque institution. Enfin, du fait du caractère moins institutionnalisé des carrières académiques en sciences de la communication, et d'un moindre cloisonnement entre les différentes écoles de pensée, les chercheurs y ont, plus souvent que dans d'autres disciplines, l'occasion de se frotter, au cours de leur parcours professionnel individuel, à des orientations épistémologiques différentes de celles auxquelles ils ont été formés ${ }^{8}$.

Une autre spécificité à relever ici réside dans l'absence d'évaluation centralisée de la recherche académique en Belgique francophone, dont le financement public est alloué aux universités sur une base non compétitive, sans lien avec les performances effectives des institutions d'enseignement supérieur ${ }^{9}$. Partant, les enjeux liés à la qualité dans l'évaluation des publications portent, d'une part, sur l'évaluation des départements et des unités de recherche, selon des critères laissés à la discrétion de chaque université. D'autre part, ces enjeux sont cruciaux dans l'évaluation de la productivité scientifique des individus, telle qu'elle s'opère au moment du recrutement, ainsi qu'à chaque moment-clé de la carrière des enseignants-chercheurs ou des chercheurs du FNRS, le Fonds national de la recherche scientifique.

Afin de tirer le meilleur parti de ces spécificités de notre terrain, et même si l'ampleur limitée de ce dernier ne nous autorisera pas à analyser systématiquement les déterminants individuels aux discours, nous avons choisi les chercheurs interviewés selon le principe de «l'échantillonnage intentionnel $\rangle^{10}$ et en respectant un critère de $« d i$ versification maximale ». Nous avons ainsi tenu compte du genre (six hommes et six femmes), de la position dans la carrière (cinq doctorants [D], un post-doctorant [PD], un chercheur qualifié du FNRS [CQ] et cinq enseignants-chercheurs [EC], l'échantillon comprenant un doyen de Faculté ainsi qu'un président de Département), de la diversité des thèmes de recherche, des institutions d'appartenance (toutes les universités belges francophones où la communication est enseignée sont représentées) et de la discipline dans laquelle le doctorat a été ou sera obtenu (neuf docteurs ou doctorants en sciences de la communication, trois docteurs issus d'autres disciplines). Enfin, il convient de relever que cinq de nos douze ré- 
pondants ont eu l'occasion de développer, à des titres divers, des accointances avec le monde de la recherche anglo-saxonne en sciences humaines, impliquant une mobilité géographique de longue durée en dehors de la francophonie.

Les entretiens avec les répondants (R1-R12) ont été retranscrits intégralement et ont fait l'objet d'un double codage. On a d'abord catégorisé les propos des chercheurs au sein de trois grandes catégories non exclusives et directement liées à notre problématique. La première rassemble les représentations des chercheurs relatives à la «qualité prescrite », à laquelle ils se sentent incités à se conformer pour être bien évalués au sein de leur institution. La deuxième regroupe les représentations relatives à la "qualité souhaitable », dont les chercheurs souhaiteraient, à titre individuel, la valorisation dans les politiques d'évaluation. La troisième catégorie concerne les descriptions que proposent les répondants de leurs activités effectives de dissémination scientifique. Un codage thématique a ensuite été opéré, visant à faire émerger les principaux thèmes ou éléments conceptuels, au sein de chacune de ces trois catégories.

Partant, l'article rendra tout d'abord compte des perceptions des chercheurs quant à la qualité prescrite en matière de publication, pour ensuite décrire le rapport ambivalent que celle-ci entretient avec la qualité souhaitable. On sera alors en mesure, dans un troisième temps, de comprendre comment les représentations du prescrit et $\mathrm{du}$ souhaitable - ainsi que les tensions éventuelles entre celles-ci - s'actualisent et se résolvent au quotidien dans les pratiques de publication des chercheurs rencontrés.

\section{Perception de la qualité prescrite}

Il faut souligner que tous les répondants partagent la perception que, pour être bien évalués dans les institutions académiques belges francophones, ainsi que dans les "bonnes " universités à l'étranger, il faut désormais produire en grande quantité, dans une "logique de performance », des articles dans des revues internationales de premier plan: «C'est clair qu'il y a une logique de production : papier, séminaire de recherche, conférences nationales et internationales, puis tu soumets ça à des revues (...) Il y a vraiment cette idée de rentabiliser absolument. ( $(\mathrm{R} 9, \mathrm{EC})$ Tous également considèrent qu'il s'agit là d'un bouleversement important dans les modes d'évaluation de la recherche, soit pour l'avoir vécu au cours de la dernière décennie, soit pour estimer que la situation du jeune chercheur actuel diffère fortement de celle de la génération qui l'a précédé.

Corollaire de cette logique productiviste, une pression à publier à un stade précoce de la carrière est également ressentie, afin de se démarquer «dans un environnement de plus en plus compétitif $»$. Il conviendrait également, pour être plus productif, de se concentrer sur quelques objets de recherche: "Dans une logique quantitative on va se concentrer sur un seul sujet, s'immerger dans la littérature puis produire un maximum d'articles dans le domaine. » (R12, EC) Enfin, si l'internationalité de la francophonie conserve de sa pertinence dans certaines institutions, c'est bien l'internationalisation à destination des espaces géographiques, ainsi que des revues anglo-saxonnes, qui est perçue comme la mieux valorisée : «Il y a une nécessité de s'inscrire dans des normes internationales: on va privilégier des 
revues classées $A$, [c'est] mieux si c'est en anglais, encore mieux si c'est aux USA » (R7, EC).

Ces représentations des chercheurs concernant les prescriptions en matière de publication sont certes congruentes avec les incitations plus générales à la productivité, à l'internationalisation et au benchmarking dans le secteur de l'enseignement supérieur. Pour autant, les répondants considèrent que la communication de la qualité prescrite en matière de publication, au niveau local de leurs départements et unités de recherche, est encore relativement peu formalisée, voire «implicite » et «peu concertée ». D'ailleurs les (jeunes) chercheurs qui briguent un mandat découvrent souvent sur le mode de l'ex post, au moment de la reconnaissance - ou non - par les jurys de leurs travaux déjà publiés, si leur production a atteint le niveau de qualité attendu.

En particulier, les critères d'excellence permettant de hiérarchiser les revues ou de les constituer en listes qualifiantes ne semblent guère explicités au niveau local : aucun des interviewés n'a reçu ou transmis d'informations formelles à ce sujet au sein de son département. Des prescriptions relatives aux classements des revues se communiquent néanmoins à l'échelle internationale - sur le mode du «bouche-à-oreille»-, dans les ateliers « carrière » et «publication» organisés en marge de conférences, les Écoles d'été, ou encore, à l'occasion de collaborations auctoriales transnationales, chaque fois qu'un co-auteur extérieur et plus expérimenté communique sa définition de la « revue de qualité ». C'est à l'international également que les critères d'évaluation de nature scientométrique seront évoqués. Au niveau local, de tels critères ne seraient pas pris en compte dans le recrutement et «ne constituent pas une notion répandue en [sciences de la] communication» (R11, EC). D'ailleurs, si les chercheurs les plus familiers avec la recherche anglo-saxonne sont bien au fait de la bibliométrie, d'autres répondants n'en connaissent pas même les principaux indicateurs et formules (Impact Factor, H-index, etc.) !

Encore largement informelles, les représentations que se font les répondants de la qualité prescrite dans l'évaluation de leurs publications constituent donc plutôt un «script», au sens conféré par la sociologie néo-institutionnaliste de «scénario [s] » cognitif, « canevas qui confère[nt] $d u$ sens à des situations données » et qui incite à se comporter d'une manière plus ou moins contraignante ${ }^{11}$. Agissant au niveau microsociologique sur les représentations des individus, le script prépare, en se structurant, l'avènement d'un discours normatif plus formalisé et mieux susceptible de persister dans le temps au niveau institutionnel.

Or, le script relatif à la qualité tel qu'il émerge des entretiens n'est pas (encore) un «script fort » : il laisse le champ libre à la manifestation d'attitudes - et de pratiques - ambivalentes à son égard. Face aux incitations à produire des articles en quantité, dans des revues de premier plan, les chercheurs rencontrés se révèlent en effet partagés entre la perception des opportunités liées au «nouveau système » et la conscience des menaces inhérentes à ce dernier. Pour en rendre compte, nous distinguerons ici entre les dimensions plutôt épistémiques de cette ambivalence, qui concernent le contenu scientifique en soi des savoirs disséminés, et ses aspects plus pragmatiques - au sens sociolinguistique du terme -, 
relatifs aux différents destinataires du texte de recherche, ainsi qu'aux effets de ce dernier sur le contexte sociétal ${ }^{12}$.

\section{Ambivalence épistémique}

La démarche de recherche particulière suivie par certains des répondants les prédispose plus naturellement à s'aligner sur les injonctions à l'internationalité extra-francophone, et à en tirer profit. En effet, la discussion savante concernant leur(s) objet(s) de recherche de prédilection - tels, par exemple, l'e-activisme, les séries télévisées ou les approches cognitivistes des TIC - est de facto menée à l'échelle internationale, et non à celle de la francophonie. Selon ces chercheurs qui participent régulièrement à la discussion savante menée dans les revues anglophones, le regard porté par les SIC francophones sur ces objets particuliers serait par «trop classique », manquant de « modèles explicatifs » et d'épistémologies alternatives, voire carrément «en retard» et peu innovant par rapport à une recherche anglosaxonne que les SHS francophones ne feraient, dans certains cas, que « traduire ». Les revues internationales constituent alors le lieu le plus souhaitable - voire, le cas échéant, le seul lieu possible ! - pour faire avancer la connaissance sur ces objets : "L'écrasante majorité de ce que je lis est publiée dans l'univers anglophone, que ce soient des publications internationales (...) ou des gens qui sont en France et qui (...) publient autant en anglais qu'en français. » (R5, CQ)

Par ailleurs, un répondant juge très souhaitable de concentrer ses activités de publication sur quelques objets de recherche spécialisés, parce que cela «renforce la légitimité » des chercheurs. Pareille concentration empêcherait en effet la dis- persion scientifique et rendrait les universitaires mieux à même de suivre la littérature concernant leur(s) objet(s) de recherche et d'enseignement : "L'idéal, c'est une charge de cours spécialisée, puis on publie dans cette spécialité !» (EC, 12R).

Pour autant, un autre chercheur revendique, au contraire, le droit à la «polygraphie » : " Toujours faire fructifier un [seul et même] article, c'est pas mon truc (...) Je ne me cantonne pas à un champ étiqueté et je m'inspire de différentes disciplines. (...) C'est là que le savoir se développe. »( $\mathrm{R} 8)$ Refusant toute approche stratégique de la publication, le même s'insurge contre les injonctions à la productivité, qu'il rend responsables du déficit qualitatif actuel de la recherche en SHS, «l'ambition première de la recherche [étant] de faire fructifier les savoirs » et pas de "privilégier la quantité à la qualité » (R8, EC).

D'autres répondants, plus nombreux, craignent que le nouveau système n'entraîne in fine un réductionnisme des modalités mêmes de production de la connaissance. Plusieurs, et parmi eux des chercheurs qui publient dans des revues anglophones, reconnaissent le « génie de la langue » française, menacé par une hégémonie anglo-saxonne. On aurait en effet plus de «finesse conceptuelle » dans sa langue maternelle, tandis que le recours à l'anglais pousserait toujours à une certaine standardisation.

Comme les chercheurs associent les objets de recherche et les quasi-paradigmes dans lesquels s'inscrivent leurs recherches à des espaces géographico-linguistiques plus ou 
moins déterminés, l'internationalisation de la recherche se pose, selon eux, en problématique épistémologique autant sinon davantage que strictement linguistique. Ainsi, il serait plus difficile de publier dans les revues internationales des recherches inscrites dans des quasiparadigmes ancrés dans les SHS francophones et qui entretiennent peu d'affinités spontanées avec les sciences sociales anglo-saxonnes, tels par exemple des travaux de narratologie. De façon plus générale, les sciences de la communication anglo-saxonnes sont perçues comme étant plus portées sur les démarches quantitatives et empiriques, et les grandes revues internationales comme moins ouvertes aux démarches de recherche qualitative. En revanche, les SIC francophones tendraient davantage à la spéculation et à l'herméneutique, et le formatage des textes publiés y serait également moins contraignant. Dans cette optique, un répondant, dont les travaux s'inscrivent résolument dans un contexte scientifique francophone, exprime ses craintes que l'internationalisation des sciences de la communication ne s'accompagne d'une forme de réductionnisme épistémologique, entraînant la disparition des modes non anglosaxons de production du savoir : «Si on arrive à une domination de l'anglais, ça modifiera nos buts de recherche: mes collègues de l'université de Leuven sont à 1000 lieues de ce qu'on fait (...). Ils ne publient qu'en anglais et dans une logique quantitative. » (R7, EC).

\section{Ambivalence pragmatique}

Exception faite du répondant «polygraphe» qui oppose quantité prescrite et qualité souhaitable (cf. supra), aucun des chercheurs rencontrés ne considère l'injonction à produire des textes de recherche comme un problème en soi. L'un d'eux ira même jusqu'à souhaiter voir implanté au sein de son département l' « esprit de valorisation » particulier qu'il attribue, pour l'avoir vécu in situ, aux SHS américaines : " Ils [les Américains] sont plus pragmatiques dans leur stratégie de recherche: une affiche dans la rue, un seul exemple peut mener à l'écriture d'un chapitre. (...) On ne se lance pas dans une recherche [aux USA] sans avoir eu des contacts préalables avec l'éditeur et s'être assuré des possibilités de la publier. » (R11, EC).

Par ailleurs, l'ensemble des répondants s'accordent à considérer l'usage de l'anglais en tant que lingua franca de la science comme une nécessité pragmatique, dès lors qu'on souhaite engager la discussion savante avec des spécialistes actifs au-delà des frontières de la francophonie. Les jeunes chercheurs reconnaissent également le rôle que la pression à publier en anglais joue dans leur insertion au sein d'un réseau international de recherche, condition sine qua non à la poursuite de la carrière (notamment pour l'obtention d'un postdoctorat). En effet, la perspective de publier en anglais pousse les doctorants à présenter une première version de leurs articles à des conférences internationales où ils pourront, le cas échéant, inciter un chercheur anglophone à collaborer avec eux sur de futurs travaux.

Face aux injonctions à produire tôt dans la carrière, les attitudes des répondants sont néanmoins très ambivalentes. D'une part, les doctorants rencontrés apprécient d'obtenir des commentaires de leurs aînés en cours de travail 
doctoral, chaque fois qu'ils soumettent un article à une revue. Cependant, quand elle est prématurée, l'exposition du jeune chercheur à ses pairs peut constituer une véritable « mise en danger »- "C'est un peu ridicule : on est amené à faire des colloques alors qu'on a encore rien à dire! » -, et lui valoir une publicité négative (R3, D). Par ailleurs, l'absence de seuil qualifiant explicite à atteindre en termes de nombre de publications est fortement regrettée à l'aube de la carrière : «Ce n'est pas écrit ; il y a un côté paradoxal de la surenchère : si mes concurrents ont un post-doc, il faut que j'en aie, pareil pour une monographie » (R2, D). Ceci aboutit à une perception paradoxale du processus d'évaluation lui-même : «Il y a un paradoxe un peu idiot : on demande à un candidat d'avoir un CV équivalent à ce qu'il aurait eu s'il avait, pour caricaturer, déjà exercé la fonction pendant 20 ans ! 》(R2).

Le risque d'un manque d'éthique lié à la survalorisation d'un seul texte de recherche et à son « recyclage » intempestif est aussi évoqué : « Je connais une chercheuse qui accumule les colloques et les conférences à l'étranger et finalement elle raconte toujours la même chose (...) ça n'a pas grand intérêt scientifique, sinon de créer des liens» (R2, D). En particulier, le principe de la «conférence in absentia »-où le papier est présenté par un chercheur qui n'en est pas forcément le co-auteur - est sujet à caution, et ne se justifierait que s'il s'agit de « travaux d'équipe avec une équipe de recherche qui se réunit et qui discute des papiers » $(\mathrm{R} 9, \mathrm{EC})^{13}$.

Certains, et parmi eux des chercheurs qui publient en anglais, regrettent que la pression à publier à l'échelle internationale ait comme corollaire la dévalorisation, pour des raisons pragmatiques plutôt qu'épistémiques, des médias scientifiques locaux ou nationaux par rapport aux revues internationales. D'où la perception également d'un certain paradoxe, à l'intérieur même des frontières de la francophonie : « Recherches en Communication [revue de l'Université Catholique de Louvain (UCL)] est bien vue en France, donc beaucoup de chercheurs français y publient (...). Le Belge est l'étranger de service; mais à l'UCL, on me dira que c'est facile de publier dans la revue de son département. Il y a une forme de dévalorisation [des publications locales] pour des raisons circonstancielles plutôt que de qualité intrinsèque. » (R2, D).

Par ailleurs, une focalisation des activités de publication sur la production d'articles dans des revues anglophones comporte le risque d'exclure de la communication scientifique internationale un certain nombre de chercheurs qui ne publient pas en anglais, et dont les travaux ne seront pas cités. Confrontés à la perception des spécificités quasiparadigmatiques évoquées plus haut, certains répondants qui publient en anglais choisiront résolument de ne référencer que des travaux anglophones, évitant ainsi de mêler dans leurs textes les différents univers de recherche : «Dans un article [en anglais] je me suis inspiré d'un chercheur dont les travaux, très connus en Allemagne, $n$ 'ont pas été traduits en anglais, et je ne l'ai pas cité. Si beaucoup ont la même attitude que moi (...), c'est handicapant pour ces auteurs [qui ne publient pas en anglais]. » (R10, PD).

Au-delà, c'est le « rôle social d'intervention 
dans la société du chercheur » qui serait remis en jeu, eu égard notamment à l'allongement des phases transitoires de type postdoctoral dans la carrière, consacrées à la publication de textes de recherche destinés aux pairs. Or : "Qui lit les revues reconnues? Les chercheurs. Donc l'impact social est presque zéro!» (R7, EC). Partant, les répondants sont unanimes pour souhaiter une meilleure reconnaissance, dans l'évaluation, de la littérature de vulgarisation scientifique ainsi que des rapports soutenant la décision politique. Ils regrettent que la qualité prescrite tende indirectement à en dévaloriser la production, en incitant à publier exclusivement dans des revues internationales de premier plan. Une situation de tension peut en résulter, entre l'envie de toucher un plus large public ou de jouer un rôle dans l'espace public d'une part, et, d'autre part, la pression à se conformer à la qualité prescrite : «[Publier dans Médiatiques, revue de vulgarisation de l'UCL], ça rentre en conflit (...) au début on le fait [mais] certains chercheurs reçoivent des consignes : " Si tu publies dans Médiatiques, c'est dommage pour toi » (...) On nous dit clairement qu'il y a intérêt à publier ailleurs (...) C'est motivant, mais si tu veux postuler au FNRS c'est pas du tout valorisé. » (R3, D).

Enfin, face au script de la qualité, certains répondants regrettent que d'autres formats médiatiques de communication scientifique, dont ils perçoivent pourtant les opportunités pragmatiques, ne soient pas davantage valorisés dans les politiques d'évaluation. Ainsi la valeur des revues uniquement disponibles en ligne - en particulier celles proposées en «Open Access », qui ouvrent le texte à un public élargi de pairs - est-elle considérée comme trop peu explicitement reconnue. Il en va de même du livre, dont la publication demeure un objectif professionnel éminemment souhaitable et qui permettrait - dans le cas d'un ouvrage collectif - d'entretenir des relations et d'échanger avec davantage de pairs spécialistes du même objet que ce n'est le cas lors de la rédaction d'un article, voire de s'ouvrir à un public élargi de lecteurs ou, dans le cas d'un manuel, de directement servir les missions d'enseignement.

\section{Bricolage avec le script}

De par leur attitude ambivalente à l'égard du script relatif à la qualité, la plupart des chercheurs rencontrés se sentent un peu "schizophrènes" ", cherchant à s'inscrire avec plus ou moins d'enthousiasme dans le nouveau système de la qualité (perçue comme) prescrite, tout en composant peu ou prou avec la qualité qu'ils jugent souhaitable : "C'est un comportement un peu schizophrène: je cours après un certain type de publics pour mon propre $C V$, alors que j'en défends un autre type en tant que directeur de publication.» (R5, CQ) Dans la pratique quotidienne, cette schizophrénie intellectuelle se résoudra régulièrement dans une " qualité en actes » composite, qui «bricole» avec les qualités prescrite et souhaitable.

Ainsi, on pourra s'efforcer d'être productif tout en se fixant ses propres règles éthiques limitant la survalorisation (cf. supra). D'autre part, la nécessité de s'internationaliser n'est pas nécessairement exclusive d'une meilleure collaboration au niveau local, mais peut lui servir de catalyseur. En effet, la définition d'axes de recherche clairs et rassembleurs au sein du département ou de l'unité 
de recherche contribue à donner une visibilité internationale à des collaborations menées d'abord au niveau local, par l'obtention d'une « masse critique » suffisante : "C'est aussi un souci de rentabilité : si tu es tout seul, tes collaborations internationales, il faut arriver à les porter!» (R9, EC). Des solutions innovantes sont par ailleurs trouvées pour combiner l'impératif de publication en langue anglaise avec la volonté de toucher un public élargi et d' ' être impliqué dans l'espace public » : «L'ambition [de la revue des Facultés universitaires Saint-Louis Brussels Studies] est de participer au débat public sur Bruxelles, d'où la nécessité d'avoir la dimension trilingue [anglais, français et néerlandais], et aussi que ça reste abordable pour le grand public, pas seulement pour les spécialistes en sciences de la communication, mais aussi pour les acteurs politiques. » (R9)

On peut, d'autre part, essayer d'être productif dans les champs de la recherche en français aussi bien qu'en langue anglaise - tenter de « jouer sur les deux tableaux »-, en tenant compte de leurs spécificités quasi-paradigmatiques respectives : « En anglais, j'ai plutôt tendance à publier des choses qui sont le résultat de données empiriques, mais je m'interdirais de mener des discussions plus théoriques. (...) Quand je soumets des articles à Recherches en Communication, ce sont des papiers spéculatifs que je ne soumettrais pas à une revue anglophone » (R5, CQ). Certains citeront des auteurs francophones dans des travaux rédigés en anglais, quitte à traduire les citations. D'autres parviendront à publier à l'international des travaux pourtant ancrés dans les traditions francophones en suscitant l'intérêt outre-Atlantique pour les singularités d'un objet « local» (par ex. le cinéma wallon) ou bien en invoquant des travaux francophones qui jouissent d'un prestige international (Ricœur, Morin, de Certeau, etc.). Enfin, la production de publications gagnera parfois à être intégrée à des activités d'enseignement et de vulgarisation: "J'étais chroniqueuse radio : ce quej'écris scientifiquement alimente mes chroniques. Parfois mes chroniques me donnent des idées, soit pour des mémoires d'étudiant, soit je les garde sous le coude, pour une publication future. » (R11, EC).

\section{Discussion : pour une normalisation non res- trictive de la qualité prescrite}

L'analyse de nos entretiens conforte donc la thèse du caractère encore informel et peu explicite de la qualité prescrite en matière d'évaluation des publications dans les sciences de la communication belges francophones, de l'ambivalence foncière des chercheurs à l'égard des prescriptions, ainsi que du mode d'intégration composite - plutôt que mécanique et unilatéral - de ces dernières dans les pratiques de dissémination scientifique. Quatre principaux facteurs explicatifs ont pu être avancés. Tout d'abord, le script de la qualité n'est pas (encore) un script fort, les critères de distinction et de hiérarchisation des revues savantes étant actuellement davantage en cours de reformulation qu'explicitement reformulés. Ensuite, les sciences de la communication étudient des objets de recherche très divers, dans la perspective de quasi-paradigmes qui ne présentent pas tous les mêmes prédispositions à la publication internationale dans des revues anglophones. Par conséquent, en fonction de ses objets de recherche de prédilection, chacun des chercheurs rencontrés perçoit en proportions variables des 
menaces et des opportunités à intégrer la qualité prescrite. Cependant, nonobstant les affinités épistémologiques plus ou moins électives de chacun avec le script de la qualité, l'ensemble des répondants se révèle conscient du risque, de nature épistémique ou plus pragmatique, qu'impliquerait une réduction exclusive des normes d'évaluation des publications à la seule qualité prescrite. Enfin, pour faire face aux menaces perçues, et plutôt que d'aligner unilatéralement leurs pratiques de publication sur le script de la qualité, les chercheurs rencontrés opèrent des « bricolages » visant à (ré)concilier, à l'échelle individuelle et à titre plus ou moins précaire, les qualités prescrite et souhaitable.

Partant, une normalisation et une formulation plus explicites - et donc plus contraignantes - de la seule qualité prescrite au sein des politiques d'évaluation des publications contribueraient sans nul doute à améliorer les performances et la place des universités belges francophones dans les benchmarks internationaux. Le danger cependant, tel qu'il émerge des attitudes ambivalentes des chercheurs rencontrés à l'égard des prescriptions, serait alors, non seulement de réduire la diversité épistémologique, linguistique et médiatique de la recherche fondamentale en sciences de la communication, mais également d'éloigner les universitaires de la production d'innovation sociale, notamment au niveau régional. Relégués dans une nouvelle tour d'ivoire, ces derniers publieraient des connaissances dûment validées à l'échelle internationale par les meilleurs spécialistes académiques, mais dénuées d'impact sociétal durable.

De façon corollaire, un repli des activités de dissémination scientifique sur la seule production exponentielle d'articles dans des revues internationales de premier plan contribuerait, au niveau institutionnel, à inscrire sur un mode éminemment paradoxal les départements de sciences de la communication dans l'espace européen de la recherche (EER). Les stratégies européennes - de Lisbonne à « Europe 2020 »-prônent en effet la mise en place d'un «triangle de la connaissance », intégrant les activités de recherche, d'enseignement ainsi que d'innovation sociale et technologique, afin d'améliorer la croissance économique et le bien-être sociétal. Or, dans le champ des sciences de la communication - dont les chercheurs produisent fort peu de brevets -, une telle intégration se conçoit difficilement sans la production et la dissémination de connaissances utiles à des publics élargis.

Face à la possible relégation des sciences de la communication à la périphérie de l'EER, comme devant les autres menaces que les entretiens menés ici ont contribué à faire émerger, il conviendrait de prolonger les recherches sur les modalités de (re)connaissance et d'incorporation de la qualité prescrite par les chercheurs, notamment par le biais d'enquêtes plus larges sur la qualité souhaitable, conduites à l'échelle internationale ${ }^{14}$. En intégrant davantage d'évidence empirique à l'élaboration des politiques d'évaluation des publications, et en valorisant plus formellement les meilleures pratiques qui ne font pas (encore) partie du script, les décideurs politiques et académiques seraient mieux à mêmes de définir des normes de qualité plus respectueuses de la variété des contenus épistémiques de la recherche en sciences de la communication, et également plus soucieuses, d'un point de vue pragmatique, 
de la diversité des contextes souhaitables de sa dissémination.

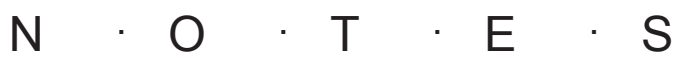

1. Voir M. Power, The Audit Society: Rituals of Verification, Oxford University Press, 1999.

2. Pour une synthèse des différents modes de conceptualisation de la qualité : L. Harvey et D. Green, " Defining Quality », in Assessment \& Evaluation in Higher Education, vol. 18, n 1, 1993, pp. 9-34.

3. Parmi l'importante bibliographie consacrée à ces évolutions, retenons ici : J. Allard, G. Haarscher et M. Puig de la Bellacas, L'université en questions. Marché des savoirs, nouvelle agora, tour d'ivoire?, Bruxelles, Labor, 2001 ; I. Bruno, À vos marques, prêts... cherchez ! La stratégie européenne de Lisbonne, vers un marché de la recherche, Editions du Croquant, coll. «Savoir/Agir », 2008 ; J. Enders, H.F. de Boer and D.F. Westerheijden (ed.), Reform of Higher Education in Europe, Sense, Rotterdam, 2011 ; E. Hazelkorn, Rankings and the Reshaping of Higher Education : the Battle for World-Class Excellence, Hundmills, Palgrave Macmillan, 2011 ; M. Lefèbvre, «L'évaluation des savoirs scientifiques : modalités et enjeux », in J. Schopfel (dir.), La publication scientifique : analyses et perspectives, Paris, Hermès, 2008, pp. 299-316 ; S. Louvel et S. Lange, «L'évaluation de la recherche : l'exemple de trois pays européens » in Sciences de la société, vol. 79, 2010, pp. 11-26 ; C. Musselin, «Vers un marché international de l'enseignement supérieur?», in Critique internationale, $\mathrm{n}^{\circ} 39$, Juin 2008, pp. 1-23.

4. Concernant l'impact général des politiques d'évaluation sur les pratiques de publication : A. Abbott, D. Cyranoski, N. Jones, B. Maher, Q. Schiermeier et R. Van Noorden, « Do Metrics Matter? », in Nature, vol. 46, n ${ }^{\circ}$, juin 2010 , pp. 860-862 ; H. F. Moed et M. Luwel, « The Influence of Policy on Scholarly 
Research : A bibliometric Point of View of the Academic Research System » in J. Hurley (ed.), Scientific research effectiveness: the organisational dimension, pp. 49-68, 2003 ; R. Vanderstraeten, « Scientific Communication : Sociology Journals and Publication Practices », in Sociology, Juin 2010, vol. 44, n³, pp. 559-576 ; voir également l'enquête du RIN (Research Information Network), Communicating Knowledge: How and why UK researchers publish and disseminate their findings, consultée sur www. rin.ac.uk/communicating-knowledge le 20 novembre 2010.

5. Voir D. Pontille et D. Torny, « The Controversial Policies of Journal Ratings: Evaluating Social Sciences and Humanities » in Research Evaluation, vol. 19, n5, pp. 347-360.

6. Voir Y. Gingras et J. Heilbron, « L'internationalisation de la recherche en sciences sociales et humaines en Europe (1980-2006) » in G. Sapiro (dir.), L'espace intellectuel en Europe : de la formation des ÉtatsNations à la mondialisation, XIX ${ }^{e}-X X I^{e}$ siècle, Paris, La Découverte, 2009, pp. 359-389.

7. On trouvera un écho privilégié de ces débats sur le carnet en ligne consacré à l'évaluation de la recherche en SHS http://evaluation.hypotheses.org/.

8. Voir F. Heinderyckx, «The academic identity crisis of the European Communication researcher », in Vestnik, vol. 6, 2008, pp. 25-29.

9. Sur les SHS en Belgique, voir le rapport du METRIS Network, consulté sur http://acceptance.all4it. com/metris/index.cfm/report/findByStructureAndCountry/25/1 le 14 juillet 2011.

10. M. Q. Patton, Qualitative evaluation and research methods, Newbury Park, Sage Publications, 1990.

11. R. Rizza, « Néo-institutionnalisme sociologique et nouvelle sociologie économique : quelles relations?», in Interventions économiques [En ligne], n³8, 2008, consulté sur http://interventionseconomiques.revues. org/292 le 20 août 2011; voir également L. Zucker, « Institutional Theories of Organizations » in Annual Review of Sociology, vol. 13, 1987, pp. 443-464 et, pour une application de la notion de script à l'orientation des carrières académiques : F. Dany, S. Louvel et A. Valette, « Academic careers: The limits of the « boundaryless approach » and the power of promotion scripts », in Human Relations, vol. 64, $\mathrm{n}^{\circ} 7$, pp. 971-996.

12. Pour un développement de l'approche épistémopragmatique appliquée au texte de recherche, voir $\mathrm{M}$. Vanholsbeeck, « Dépôt numérique des publications et renouveau des relations entre les acteurs de la recherche "), in S. Proulx et A. Klein (dir.), Connexions : Communication numérique et lien social, Namur, Presses universitaires de Namur, sous presses.

13. Sur ces dérives liées à la surproductivité publicationnelle : P. Lawrence, « Lost in publication : how measurement harms science », in Ethics in Science and Environmental Politics, n8, 2008, pp. 9-11.

14. L'auteur a participé à la réalisation d'une telle enquête, concernant les sciences de la communication, dont les résultats devraient être prochainement publiés. 


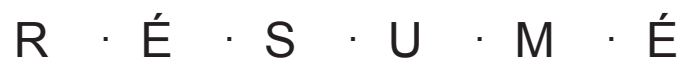

Cet article investigue, à partir d'entretiens compréhensifs, comment des chercheurs en sciences de la communication de Belgique francophone perçoivent et intègrent à leurs pratiques de dissémination scientifique la notion de qualité, telle que la sollicitent les dispositifs récents, et fortement controversés, visant à évaluer les publications en sciences humaines. Trois présupposés sousjacents aux débats sur l'évaluation seront ainsi questionnés : celui du caractère explicite et formalisé des prescriptions actuelles en matière de qualité, celui de la dichotomie des attitudes des chercheurs à leur égard (adhésion vs résistance), et celui de la nature unilatérale et mécanique de l'impact de l'évaluation sur les pratiques. Si les répondants associent prioritairement la «qualité prescrite » à la production d'articles au sein de revues internationales de premier plan, l'analyse révèle en effet que les critères de distinction et de hiérarchisation de ces dernières ne font pas l'objet de critères explicites. Largement informel, le « script » de la qualité suscite chez les chercheurs des représentations foncièrement ambivalentes, et la perception de menaces autant que d'opportunités. Cette ambivalence semble se résoudre au quotidien dans des pratiques qui « bricolent » avec les prescriptions et qui - du fait, notamment, de leur potentiel en innovation sociale - gagneraient à être plus valorisées dans les politiques d'évaluation.

\section{Abstract}

This article is based on twelve in-depth interviews with communication scholars working within the French-speaking part of Belgium. It investigates how they perceive and integrate to their publishing practices the somehow controversial concept of quality, as it is used nowadays in order to evaluate the scholarly publications within the humanities and social sciences. Three specific assumptions underlying the recent discussions around the evaluation will be questioned here as well, id est the explicit and formalized character of the current requirements for quality, the dichotomy of the researchers' attitudes regarding those requirements between full acceptance and stubborn resistance and the unilateral nature of the impact of the evaluation on the publishing practices. It appears indeed that although the respondents primarily link the «prescribed quality» with the production of articles in international top journals, the criteria that should be used in order to prioritize those journals and to differentiate between them are not explicitly formulated. Largely informal, the «script» of quality relating to the evaluation of scholarly publications gives rise to deeply ambivalent representations on the side of the interviewed researchers, and to perceptions of threats as well as of opportunities. This ambivalence seems to resolve itself in everyday practices that «tinker» with the prescribed quality requirements. These kinds of daily tinkering practices should indeed be more valued by policy makers and academic authorities, not the least because of their high potential in societal innovation. 